\section{Kidney \\ Blood Pressure Research}

\title{
Prediction of ESRD in IgA Nephropathy Patients from an Asian Cohort: A Random Forest Model
}

\author{
Yexin Liu Yan Zhang $^{\mathrm{b}} \quad$ Di Liu $^{\mathrm{a}} \quad \mathrm{Xia}_{\text {Tan }}^{\mathrm{a}} \quad$ Xiaofang Tang ${ }^{\mathrm{a}} \quad$ Fan Zhang ${ }^{\mathrm{a}}$ \\ Ming Xia ${ }^{a}$ Guochun Chen ${ }^{a}$ Liyu He ${ }^{a}$ Letian Zhou ${ }^{a}$ Xuejing Zhu ${ }^{a}$ \\ Hong Liu ${ }^{a}$
}

aDepartment of Nephrology, the Second Xiangya Hospital, Central South University, Key laboratory of

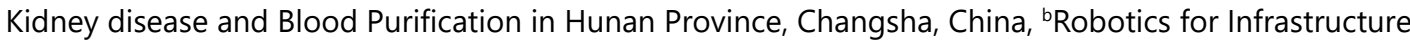
Management Team, RIKEN Center for Advanced Intelligence Project, Tokyo, Japan

\section{Key Words}

IgA nephropathy $(\operatorname{lgAN}) \cdot$ prognosis - End-stage renal disease(ESRD) $\cdot$ Random forest model - Logistic regression - pathological grading • Complement • Estimated glomerular filtration rate (eGFR)

\begin{abstract}
Background/Aims: There is an increasing risk of end-stage renal disease (ESRD) among Asian people with immunoglobulin A nephropathy (IgAN). A computer-aided system for ESRD prediction in Asian IgAN patients has not been well studied. Methods: We retrospectively reviewed biopsy-proven IgAN patients treated at the Department of Nephrology of the Second Xiangya Hospital from January 2009 to November 2013. Demographic and clinicopathological data were obtained within 1 month of renal biopsy. A random forest (RF) model was employed to predict the ESRD status in IgAN patients. All cases were initially trained and validated, taking advantage of the out-of-bagging(OOB) error. Predictors used in the model were selected according to the Gini impurity index in the RF model and verified by logistic regression analysis. The area under the receiver operating characteristic(ROC) curve (AUC) and F-measure were used to evaluate the RF model. Results: A total of 262 IgAN patients were enrolled in this study with a median follow-up time of 4.66 years. The importance rankings of predictors of ESRD in the RF model were first obtained, indicating some of the most important predictors. Logistic regression also showed that these factors were statistically associated with ESRD status. We first trained an initial RF model using gender, age, hypertension, serum creatinine, 24-hour proteinuria and histological grading suggested by the Clinical Decision Support System for IgAN (CDSS, www.lgAN.net). This 6-predictor model achieved a F-measure of 0.8 and an AUC of $92.57 \%$. By adding Oxford-MEST scores, this model outperformed the initial model with an improved AUC (96.1\%) and F-measure (0.823). When C3 staining was incorporated, the AUC
\end{abstract}




\section{Kidney Blood Pressure Research}

Kidney Blood Press Res 2018;43:1852-1864

\begin{tabular}{l|l}
\hline DOI: $10.1159 / 000495818$ & (c) 2018 The Author(s). Published by S. Karger AG, Basel
\end{tabular}

Published onlıne: 8 December 2018

www.karger.com/kbr

1853

was $97.29 \%$ and F-measure increased to 0.83 . Adding the estimated glomerular filtration rate (eGFR) improved the AUC to $95.45 \%$. We also observed improved performance of the model with additional inputs of blood urea nitrogen (BUN), uric acid, hemoglobin and albumin. Conclusion: In addition to the predictors in the CDSS, Oxford-MEST scores, C3 staining and eGFR conveyed additional information for ESRD prediction in Chinese IgAN patients using a RF model.

(C) 2018 The Author(s)

Published by S. Karger AG, Basel

\section{Introduction}

Immunoglobulin A nephropathy (IgAN) is the most common primary glomerular kidney disease in China and worldwide $[1,2]$. The clinical outcome of this disease ranges from asymptomatic hematuria to progressive renal failure and even end-stage renal disease (ESRD) [3-5]. Approximately 20-40\% of affected patients will reach ESRD within 10-20 years of diagnosis [6, 7]. Early prediction of ESRD in IgAN patients is valuable.

Methods have been developed to assist in predicting ESRD in IgAN patients. A single influential risk factor is of good value for differentiating the patients who are at great risk of ESRD [8-10]. Intuitively, a combination of multiple risk factors can be more powerful than one risk factor, and researchers have built a few models integrating multiple factors $[3,4$, 11]. A new prognostic absolute renal risk (ARR) model uses hypertension, proteinuria $\geq 1$ $\mathrm{g} / 24 \mathrm{~h}$ and severe histopathological renal lesions to predict ESRD or death in IgAN patients [11]. The ARR model employs a risk stratification score ranging from 0-3 points, which is practical for clinical use. However, this model does not integrate the initial estimated glomerular filtration rate (eGFR) or serum creatinine, which are strong predictors for poor renal outcome in IgAN patients $[3,4,12]$.

Recently, an artificial neural network (ANN) model has been applied to IgAN, and it showed superior predictive accuracy compared to the ARR model [13]. The trained ANN model has been developed into an online Clinical Decision Support System (CDSS) for quantitative risk assessment of ESRD and the timing of ESRD in IgAN patients (www.IgAN. net). The CDSS integrates 6 predictors including initial serum creatinine to predict ESRD. However, the eGFR is not included. The ANN model is trained and evaluated based on cohorts from different populations. However, the Asian population is relatively smaller than the other populations. Therefore, general application of the CDSS, especially in Asian patients, might need further validation and consideration. Of note, the criteria for severe histopathological renal lesions varied among these studies, which also hindered the general application of the prediction models. Thus, we are interested in whether the MEST criteria of the Oxford classification, eGFR and other indexes can provide further prognostic information. Moreover, an ANN is a black-box model, namely, it is difficult to interpret how a given model makes use of the predictive factors to determine whether each individual is at risk for ESRD or not at certain time points.

Motivated by the above mentioned problems, we propose the use of a random forest (RF) [14] model for ESRD prediction based on a Chinese cohort in this paper. RF models have several advantages. For instance, a RF is an ensemble of decision tree models, which are not black-box models and can be easily interpreted. Moreover, predictive factors, such as hypertension, proteinuria, eGFR, MEST and C3 staining, which refer to the severity at disease onset, are interconnected, and their individual contributions to the overall risk are not easily evaluated from a statistical perspective. This problem can be addressed by the importance ranking function of RFs, from which we can obtain an importance score for each factor reflecting its individual contribution. According to importance scores, we attempted to explore the predictive value of clinicopathological parameters for predicting ESRD thus laying the foundation for a more suitable predictive model for Asian IgAN patients. 


\section{Kidney Blood Pressure Research}

Liu et al.: ESRD Prediction in IgAN Patients via a Random Forest Model

\section{Materials and Methods}

\section{Study cohorts}

Patients with biopsy-proven IgAN from the Department of Nephrology at the Second Xiangya Hospital were first screened from January 2009 to November 2013. The exclusion criteria were as follows (1) age $<14$ years old or $>65$ years old, (2) secondary IgA deposition, (3) eGFR $<15 \mathrm{ml} / \mathrm{min}$ per $1.73 \mathrm{~m}^{2}$, (4) coexistence of other renal diseases and (5) less than 7 glomeruli in a renal tissue section. We also excluded those patients with missing eGFR, blood pressure, and proteinuria data at the time of biopsy. Two hundred and sixty-two IgAN patients-were enrolled and followed-up for a median of 4.66 years to formulate this model. IgAN was diagnosed and defined on immunofluorescence microscopy by the presence of predominant mesangial IgA deposits with or without other immunoglobulins. The eGFR was calculated by the Modification of Diet in Renal Disease (MDRD) study equation $[8,15]$. The end point was ESRD, which was defined as an eGFR $<15$ $\mathrm{ml} / \mathrm{min}$ per $1.73 \mathrm{~m}^{2}$ or the need for renal replacement therapy by dialysis (maintenance hemodialysis, maintenance peritoneal dialysis) or kidney transplantation. All studies were conducted in accordance with guidelines of the Declaration of Helsinki and with approval of Ethic Committee at the Xiangya Medical School of Central South University.

\section{Evaluation of clinicopathological parameters}

Clinical data, such as serum creatinine and urine protein, were obtained within 1 month of renal biopsy. The eGFR was calculated with an abbreviated MDRD equation, as mentioned above. Blood pressure was displayed in the form of systolic blood pressure/ diastolic pressure, and the mean arterial pressure and the number of patients who had a blood pressure over 140/90mmHg were recorded. Twenty-four hour urine samples were collected to detect urine protein excretion. The use of renin-angiotensin system (RAS) inhibitors including angiotensin-converting enzyme inhibitors (ACEIs), angiotensin receptor blockers (ARBs), or both was recorded. Immunosuppressants included corticosteroids alone or with the use of cytotoxic agents.

Histological lesions were recorded by two experienced renal pathologists who were blinded to the clinical data and patient outcomes. According to the classification used in the ANN model [13], histological grading was defined as: G1 (mild) in subjects with minimal or minor lesions, G2 (moderate) in patients with diffuse proliferative or focal segmental glomerulonephritis and G3 (severe) in those with sclerotic and advanced chronic renal lesions. The details of other pathological data were also reviewed according to the Oxford classification criteria for IgAN [16]. MEST scores were reported as follows: M0/M1 was defined as the absence or presence, respectively, of $50 \%$ of glomeruli showing hypercellularity, E0/E1 was defined as the absence or presence, respectively, of endocapillary hypercellularity, S0/S1 was defined as the absence or presence, respectively, of segmental sclerosis or tuft adhesions, and T0/T1/T2 was defined as the degree of tubular atrophy or interstitial fibrosis $(<25 \%, 26-50 \%,>50 \%$, respectively). In addition, global and segmental glomerulosclerosis were calculated as the proportion of involved sclerotic glomeruli divided by the total number of glomeruli. Interstitial inflammatory lesions were assessed by the presence or absence of such lesions. Interstitial fibrosis; and tubular atrophy were evaluated as the degree of the affected cortical area, namely, mild, moderate and severe $(<25 \%, 25-49 \%,>50 \%$ respectively).

\section{Random forest model}

A RF model is an ensemble of many decision tree models, each of which is characterized by a tree-like structure. Fig. 1 demonstrates an example of a decision tree. The decision tree directs input samples from the root node to one of the leaf nodes. When a case is inputted into the decision tree, the root node first checks the value of eGFR of the input case. If the eGFR $>50$, the model further checks the age of the patient on the left child node. If the eGFR is less than 50 , the case proceeds to the right node, i.e., checking gender and proteinuria. Recursively, the case flows into one of the leaves in the diagram, resulting in a prediction. The prediction result of a random forest model is given by the majority vote of results predicted by the decision trees.

It is common to split the data into a training set and a validation set, which would reduce the number of patients used for training, and hence possibly resulting in an inferior prediction accuracy. In the random forest model, it is not required to split the dataset, and all patients can be used for training. The bootstrap 


\section{Kidney Blood Pressure Research}

aggregating (bagging) technique [17] used by the random forest enables us to obtain a so-called out-ofbagging (OOB) error on the training dataset. As empirically shown in [18], the OOB error on the training dataset is an unbiased estimation of generalization error (i.e. testing error on the unseen testing dataset) of the random forest.

\section{Statistical analysis}

Data in normal distribution are displayed as the mean \pm standard deviation (SD). Other continuous measures are reported as the median and interquartile range. Categorical data are presented as the form of $\mathrm{n}(\%)$. Odds ratios (OR= EXP (B)) and 95\% confidence intervals (95\%CIs) from the binary logistic regression analysis were used to assess and validate the importance of different variables. Statistical significance was determined as a $\mathrm{P}$ value $<0.05$. SPSS 19.0 software was used for statistical analysis.

To predict the ESRD status at a median 4.66 years of follow-up, the model was initially trained and validated on 262 cases, taking advantage of OOB error. The performance of the final model was presented as an F-measure. Besides, the area under the receiver operating characteristic (ROC) curve (AUC) was used to measure the performance of the integrated model.

\section{Results}

Clinical and pathological characteristics of the population

From January 2009 to November 2013, 345 IgAN patients of 450 patients with biopsy proven IgAN; were eligible according to the exclusion criteria. Ultimately, 262 of 345 eligible patients were followed-up and completely available for final analysis (Fig. 2). In our cohort, $7.35 \%$ of the 262 IgAN patients reached the endpoint within a median followup year of 4.66 years.

The mean age at IgAN diagnosis was 32.71 years (SD, 11.83 years), and $47.3 \%$ of patients were males. Hypertension was observed in $66(25.2 \%)$ patients. The mean eGFR of the patients enrolled in this cohort was $92.58 \mathrm{~mL} /$ $\min / 1.73 \mathrm{~m}^{2}$ (SD, $32.62 \mathrm{~mL} / \mathrm{min} / 1.73 \mathrm{~m}^{2}$ ). Other demographic, clinical, and laboratory data of the IgAN patients are shown in Table 1.

None of the patients were receiving medication at the time of renal biopsy. After diagnosis, 143 (63.6\%) patients received RAS inhibitors. During the course, $45(19.9 \%)$ patients received corticosteroids or other immunosuppressants including cyclophosphamide, tacrolimus and mycophenolate mofetil, as appropriate. Judging from the protective role of RAS inhibitors against kidney disease progression [19], we presented the data of the proportion of patients who were treated with RAS inhibitors in each group. There was no significant difference in the proportion of patients who were treated with RAS
Table 1. The demographic, clinical, laboratory data and treatment of the IgAN patients. Abbreviations: MAP: mean arterial pressure, eGFR: estimated glomerular filtration rate, BUN: blood urea nitrogen, RAS: renin-angiotensin system. Steroids include prednisolone, methylprednisolone and others; Immunosuppressants include cyclophosphamide, tacrolimus, mycophenolate mofetil and others. Conversion factors for units: serum creatinine in $\mathrm{mg} / \mathrm{dL}$ to $\mu \mathrm{mol} / \mathrm{L}, \times 88.4$

\begin{tabular}{|c|c|}
\hline Demographic/clinical characteristics & $\mathrm{N}=262$ \\
\hline \multirow{2}{*}{ Gender , n, \% } & $124(47.3)$ \\
\hline & $138(52.7)$ \\
\hline Age, year & $32.71 \pm 11.83$ \\
\hline Course of Disease, month & $19.49 \pm 33.29$ \\
\hline Macrohematuria, n,\% & $39(14.9)$ \\
\hline Systolic pressure, $\mathrm{mmHg}$ & $126.23 \pm 15.95$ \\
\hline Diastolic pressure, $\mathrm{mmHg}$ & $79.96 \pm 11.78$ \\
\hline MAP & $95.31 \pm 12.17$ \\
\hline Hypertension, n,\% & $66(25.2)$ \\
\hline \multicolumn{2}{|l|}{ Laboratory data } \\
\hline Hemoglobin, g/L & $128.62 \pm 19.73$ \\
\hline Albumin, g/L & $37.19 \pm 8.73$ \\
\hline Serum Creatinine, $\mu \mathrm{mol} / \mathrm{L}$ & $90.38 \pm 45.58$ \\
\hline $\mathrm{eGFR}, \mathrm{mL} / \mathrm{min} / 1.73 \mathrm{~m}^{2}$ & $92.58 \pm 32.62$ \\
\hline $\mathrm{BUN}, \mathrm{mmol} / \mathrm{L}$ & $8.07 \pm 27.99$ \\
\hline Uric Acid, $\mu \mathrm{mol} / \mathrm{L}$ & $349.19 \pm 110.18$ \\
\hline Triglyceride, mmol/L & $2 \pm 2.15$ \\
\hline Cholesterol, mmol/L & $5.37 \pm 2.47$ \\
\hline Serum IgG, g/L & $10.52 \pm 3.84$ \\
\hline Serum IgA, g/L & $2.64 \pm 1.18$ \\
\hline Serum IgM, g/L & $2.87 \pm 20.61$ \\
\hline Serum C3, g/L & $1.02 \pm 0.29$ \\
\hline Serum C4,g/L & $0.24 \pm 0.15$ \\
\hline Urine protein, $\mathrm{g} / 24 \mathrm{~h}$ & $1.54 \pm 2.26$ \\
\hline Urine $\mathrm{RBC}, \times 10^{3} / \mathrm{ml}$ & $47.5(12.5,107.5)$ \\
\hline \multicolumn{2}{|l|}{ Treatments } \\
\hline RAS inhibitors, $n, \%$ & $143(63.6)$ \\
\hline Steroids or Immunosuppressants & $45(19.9)$ \\
\hline
\end{tabular}




\section{Kidney \\ Blood Pressure Research}

inhibitors between the non-ESRD group and the ESRD group $(64.5 \%$ vs $50.0 \%$, $\mathrm{p}=0.276$ ). The proportion of patients who were treated with corticosteroids or immunosuppressants in the non-ESRD group (19.4\%) was also identical to that in the ESRD group (26.7\%) ( $\mathrm{p}=0.498)$.

In terms of pathological changes, the crescent component and scores of mesangial hypercellularity(M), endocapillary hypercellularity(E), segmental glomerulosclerosis(S), and tubular atrophy/interstitial fibrosis(T) are illustrated in Table 2, accompanied by the severities of glomerulosclerosis, and tubulointerstitial lesions. Additionally, histological lesions were scored with three grades according to the classification used in the ANN model [13] as part of the CDSS for IgAN (www.IgAN.net): G1 (mild) , G2 (moderate) and G3 (severe). Finally, immunostainings for various immunoglobulins and complement components were analysed.

\section{Predictors of ESRD status}

To identify crucial predictors of ESRD, we employed two methods for analysis.

First, we established the importance ranking by the RF method. To compute the importance of each predictive factor, we trained a RF model using all the factors. In our trained RF model, each node uses a factor to direct samples to one of its two descendent nodes, as shown in Fig. 1. The contribution (importance) of this factor on this node is computed by the decrease of Gini impurity index,

$$
\mathrm{I}=G_{p}-G_{l}-G_{r}
$$

where $G_{p}, G_{l}, G_{r}$ are the Gini impurity index of this node and its two descendent nodes respectively. Gini impurity index is computed by

$$
G_{p}=p_{+}\left(1-p_{+}\right)+p_{-}\left(1-p_{-}\right)
$$

where $p_{+}, p_{-}$denotes the ratio of positive samples (patients who reached ESRD) and negative samples in this node. Analogously, $G_{l}, G_{r}$ can be computed in the same way. As we can see from equation (2), when the samples in this node are all positive or all negative samples, Gini impurity index is minimized. It reflects the impurity of the labels of samples in this node. In Equation (1), a large I indicates a large decrease of impurity from this node to its child nodes, which reflects that the predictor used in this node is important for discriminating positive samples from negative samples. We sum up the decreases of Gini impurity index over all nodes using this predictor to obtain the importance of this predictor. The importance ranking results are shown in Fig. 3.

Table 2. The Pathological characteristics of the IgAN th. * Histological grading is classified according namely, in Clinical Decision Support System for IgAN

\begin{tabular}{|c|c|c|}
\hline Pathological characteristics & & $\mathrm{N}$ \\
\hline Oxford classifications & & $\mathrm{N}=262$ \\
\hline \multirow{2}{*}{ Mesangial Hypercellularity, n,\% } & M0 & $244(93.1)$ \\
\hline & M1 & $18(6.9)$ \\
\hline \multirow{2}{*}{ Endocapillary Hypercellularity, n,\% } & E0 & $235(89.7)$ \\
\hline & E1 & $27(10.3)$ \\
\hline \multirow{3}{*}{ Segmental glomerulosclerosis, n, $\%$} & So & $101(38.5)$ \\
\hline & S1 & $161(61.5)$ \\
\hline & T0 & $162(61.8)$ \\
\hline \multirow[t]{2}{*}{ Tubular atrophy / interstitial fibrosis, n, $\%$} & $\mathrm{~T} 1$ & $76(29.0)$ \\
\hline & $\mathrm{T} 2$ & $24(9.2)$ \\
\hline \multicolumn{3}{|l|}{ Histological grading * } \\
\hline Mild & & $27(10.3)$ \\
\hline Moderate & & $142(54.2)$ \\
\hline Severe & & $93(35.5)$ \\
\hline \multicolumn{3}{|l|}{ Immunofluorescence staining } \\
\hline & $1+$ & $39(14.89)$ \\
\hline \multirow[t]{2}{*}{ IgA } & $2+$ & $129(49.24)$ \\
\hline & $\geq 3+$ & $94(35.87)$ \\
\hline IgG & Present & $69(26.2)$ \\
\hline \multirow[t]{2}{*}{ IgM } & Present & $84(32.06)$ \\
\hline & $1+$ & $60(22.9)$ \\
\hline \multirow[t]{2}{*}{$\mathrm{C} 3$} & $2+$ & $46(17.56)$ \\
\hline & $\geq 3+$ & $10(3.82)$ \\
\hline C1q & Present & $12(4.6)$ \\
\hline Fibrinogen & Present & $11(4.2)$ \\
\hline \multicolumn{3}{|l|}{ Other histological features } \\
\hline Crescents, n, \% & & $52(19.8)$ \\
\hline Global glomerulosclerosis, $\%$ & & $15.44 \pm 17.93$ \\
\hline Segmental glomerulosclerosis, $\%$ & & $9.9 \pm 11.75$ \\
\hline \multirow{3}{*}{ Interstitial inflammation, $n, \%$} & Absent & $51(19.5)$ \\
\hline & Present & $211(80.5)$ \\
\hline & Mild & $162(61.8)$ \\
\hline \multirow[t]{3}{*}{ Interstitial fibrosis, n,\% } & Moderate & $76(29)$ \\
\hline & Severe & $24(9.2)$ \\
\hline & Mild & $160(61.1)$ \\
\hline \multirow[t]{2}{*}{ Tubular atrophy, n,\% } & Moderate & $79(30.2)$ \\
\hline & Severe & $23(8.8)$ \\
\hline
\end{tabular}
(CDSS, www.IgAN.net) 


\section{Kidney Blood Pressure Research}

Fig. 1. An illustration of decision tree model for ESRD prediction in IgAN. A random forest model is an ensemble of many decision tree models.

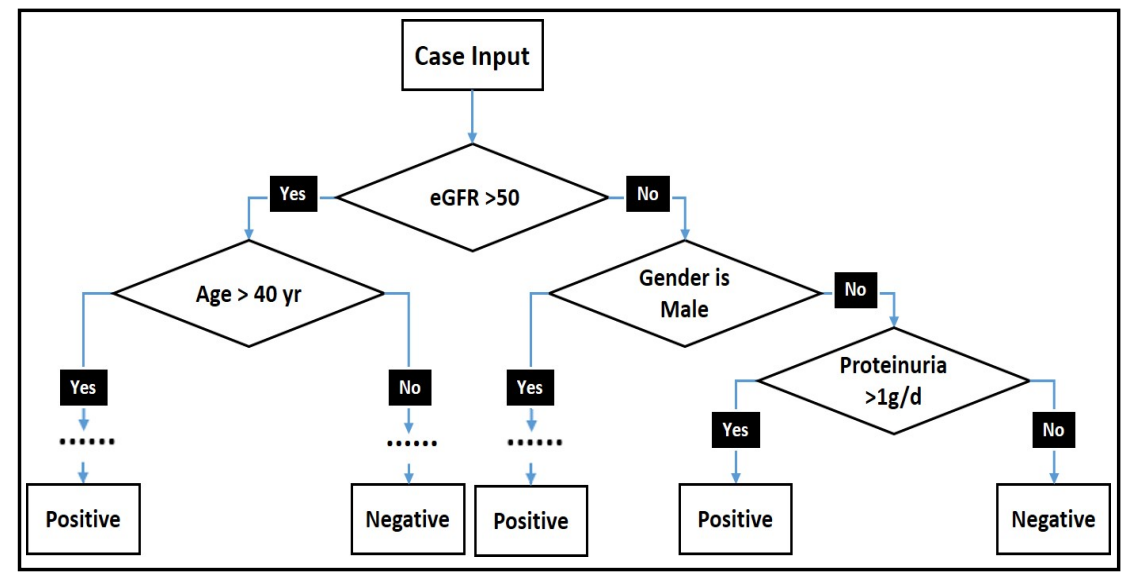

Fig. 2. Enrollment and follow-up of IgAN patients in our cohorts. IgAN: IgA nephropathy.
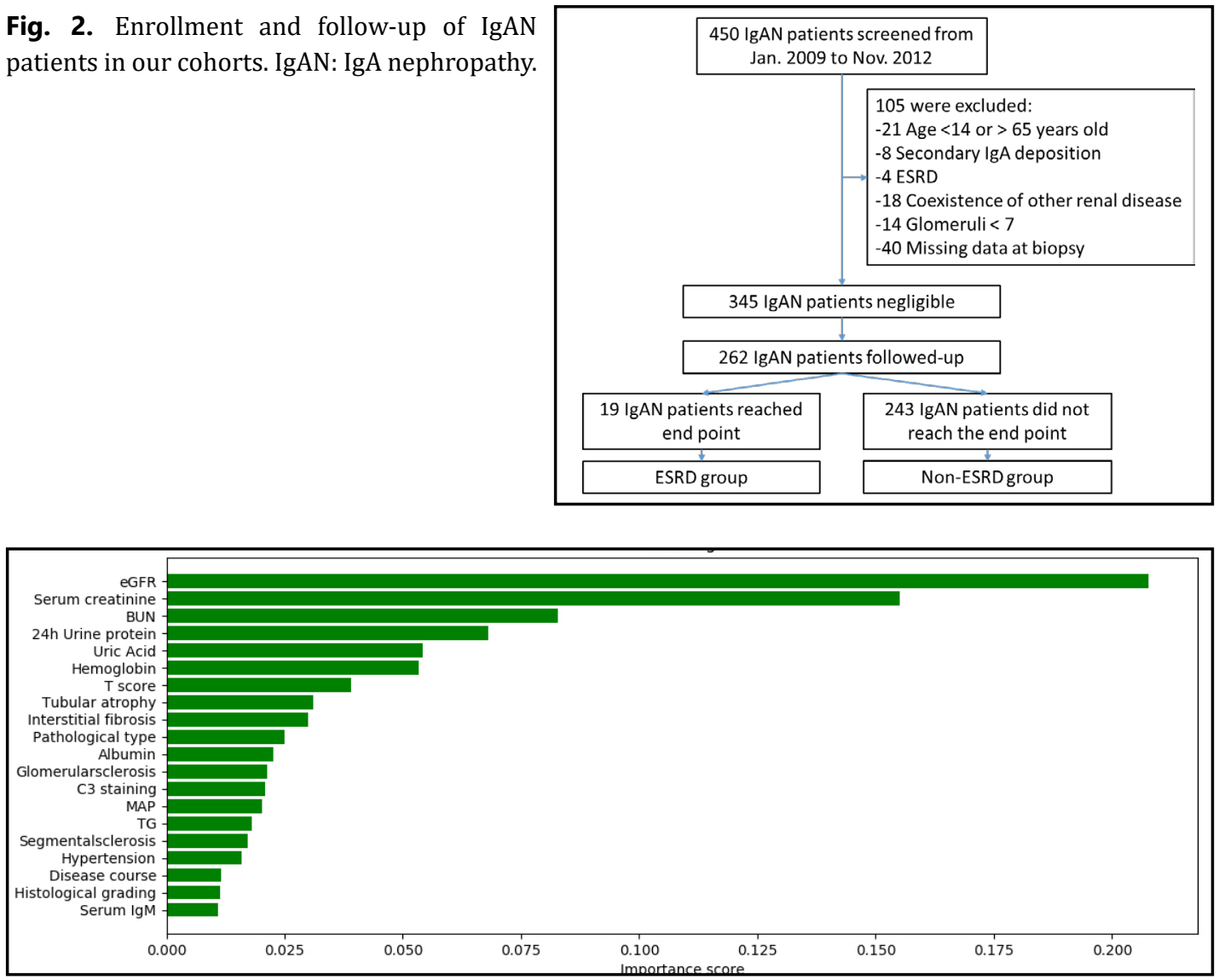

Fig. 3. Contribution of predictors of ESRD in IgAN patients (Top 20 displayed) eGFR: estimated glomerular filtration rate, BUN: blood urea nitrogen,TG: Triglyceride.

Second, a statistical method was applied to assess and verify the importance of each predictive factor. The univariate logistic regression analysis is displayed in Table 3. Patients who progressed to ESRD had a relatively low eGFR, increased serum creatinine, serum IgM, BUN, and uric acid, decreased hemoglobin and albumin levels, and increased amounts of 24-h urine protein levels at the time of renal biopsy. In terms of pathological features, S and T scores, concomitant with global and segmental glomerulosclerosis, histological grading and C3 staining were associated with poor renal survival in IgAN patients. 


\section{Kidney Blood Pressure Research}

Kidney Blood Press Res 2018;43:1852-1864

\begin{tabular}{l|l}
\hline DOI: $10.1159 / 000495818$ & (c) 2018 The Author(s). Published by S. Karger AG, Basel
\end{tabular}

Published online: 8 December 2018 www.karger.com/kbr

1858
Predictors with large importance scores were roughly in accordance with those showing significant ORs in the logistic regression analysis. Among the various predictors, the eGFR, serum creatinine levels, 24-h urine protein levels, BUN, uric acid, C3 staining, hemoglobin and albumin levels, hypertension and $\mathrm{T}$ score in the Oxford classification were of great importance in both analyses. The combination of these predictors may be more useful for stratifying patients who were likely to reach the endpoint at the time of renal biopsy. Therefore, we investigated into these factors equally for predicting ESRD status.

\section{Performance of the RF model}

We used the entire dataset including all 262 patients as a training set without a validation set by taking advantage of the OOB error. We first trained an initial $\mathrm{RF}$ model using the following predictors: gender, age, hypertension, 24-h urine protein levels and histological grading, which were the same as those used in the ANN model [13]. This RF model with 6 predictors achieved an F-measure of 0.8 and the an AUC of $92.57 \%$ (Fig. 4, area under the yellow line).

Then, according to the order of the importance rankings (Fig. 3), we trained the model by adding the predictors with considerable contributions and evaluated the performance of the model (Table 4). After integrating the Oxford-MEST scores, the model outperformed the initial model with an improved AUC (96.1\%) and F-measure (0.823) (Fig. 4A). When the model incorporated C3 staining as an additional input, the AUC was 97.29\% and F-measure increased to 8.3(Fig. 4B). However, when MEST scores and C3 staining were both added to the model, the AUC was not improved further (Fig. 4C). Addition of the eGFR also increased the AUC to $95.45 \%$ (Fig. 4D). Again, we found better performance with the addition of the predictors of BUN, uric acid, hemoglobin and albumin (Fig. 4E-4H).
Table 3. Univariate logistic regression for ESRD in IgAN. OR= odds-ratio, $\mathrm{CI}$ = confidence interval, eGFR= estimated glomerular filtration rate. Conversion factors for units: serum creatinine in $\mathrm{mg} / \mathrm{dL}$ to $\mu \mathrm{mol} / \mathrm{L}, \times 88.4$

\begin{tabular}{lcc}
\hline Predictor & OR(95\%CI) & P-value \\
\hline Gender & $1.26(0.49-3.20)$ & 0.6 \\
Age & $0.50(0.21-1.18)$ & 0.1 \\
Macrohematuria & $1.31(0.36-4.82)$ & 0.7 \\
Hypertension & $6.00(2.25-15.99)$ & $<0.001$ \\
MAP & $3.44(0.74-15.98)$ & 0.1 \\
Hemoglobin & $0.20(0.08-0.54)$ & 0.001 \\
Albumin & $0.41(0.23-0.76)$ & 0.004 \\
Serum Creatinine & $13.07(6.07-28.15)$ & $<0.001$ \\
eGFR & $0.11(0.05-0.24)$ & $<0.001$ \\
BUN & $12.61(4.33-36.68)$ & $<0.001$ \\
Uric Acid & $5.24(1.69-16.28)$ & 0.003 \\
Triglyceride & $1.22(0.48-3.1)$ & 0.7 \\
Cholesterol & $1.22(0.50-3.02)$ & 0.7 \\
Serum IgG & $0.99(0.43-2.27)$ & 0.9 \\
Serum IgA & $0.64(0.27-1.51)$ & 0.3 \\
Serum IgM & $3.86(1.72-8.69)$ & 0.001 \\
Serum C3 & $0.67(0.31-1.45)$ & 0.3 \\
Serum C4 & $2.95(0.75-11.64)$ & 0.1 \\
24h Urine protein & $3.04(1.84-4.99)$ & $<0.001$ \\
Pathological type & $1.81(1.33-2.45)$ & $<0.001$ \\
IgA staining & $1.36(0.79-2.34)$ & 0.3 \\
IgG staining & $1.56(0.93-2.62)$ & 0.09 \\
IgM staining & $1.59(0.85-2.98)$ & 0.1 \\
C3 staining & $1.71(1.08-2.70)$ & 0.02 \\
Global glomerulosclerosis,\% & $4.66(2.59-8.40)$ & $<0.001$ \\
Segmental glomerulosclerosis,\% & $4.24(2.18-8.24)$ & $<0.001$ \\
Mesangial Hypercellularity(M) & $1.67(0.35-7.87)$ & 0.5 \\
Endocapillary Hypercellularity(E) & $1.71(0.47-6.30)$ & 0.4 \\
Segmental Glomerulosclerosis(S) & $3.61(1.02-12.70)$ & 0.05 \\
Tubular atrophy/ interstitial fibrosis(T) & $5.68(2.84-11.37)$ & $<0.001$ \\
Crescents & $1.98(0.71-5.48)$ & 0.2 \\
Histological Grade & $4.14(1.61-10.64)$ & 0.003 \\
Tubular atrophy, \% & $5.91(2.91-12.01)$ & $<0.001$ \\
Interstitial fibrosis,\% & $5.68(2.84-11.37)$ & $<0.001$ \\
Interstitial inflammation, \% & $4.66(0.61-35.78)$ & 0.1 \\
\hline & & \\
& & \\
& &
\end{tabular}

Table 4. Performance comparison for the prediction on ESRD status with different predictors. * Six predictors are age, gender, hypertension, serum creatinine, 24-hour proteinuria, and histological grading by the ANN model, namely, Clinical Decision Support System for IgAN (CDSS, www.IgAN. net). Abbreviations: ANN: artificial neural networks eGFR: estimated glomerular filtration rate, BUN: blood urea nitrogen, UA: uric Acid

\begin{tabular}{lcc}
\hline Predictor & F-measure & AUC \\
\hline Six predictors proposed in the ANN model* & 0.8 & 0.9257 \\
Six predictors plus MEST & 0.8235 & 0.9610 \\
Six predictors plus C3 staining & 0.83 & 0.9729 \\
Six predictors plus C3 staining \&MEST & 0.8235 & 0.9702 \\
Six predictors plus eGFR & 0.777 & 0.9545 \\
Six predictors plus BUN & 0.777 & 0.9526 \\
Six predictors plus UA & 0.7368 & 0.9538 \\
Six predictors plus Albumin & 0.7777 & 0.9657 \\
Six predictors plus Hemoglobin & 0.7879 & 0.9594 \\
\hline
\end{tabular}




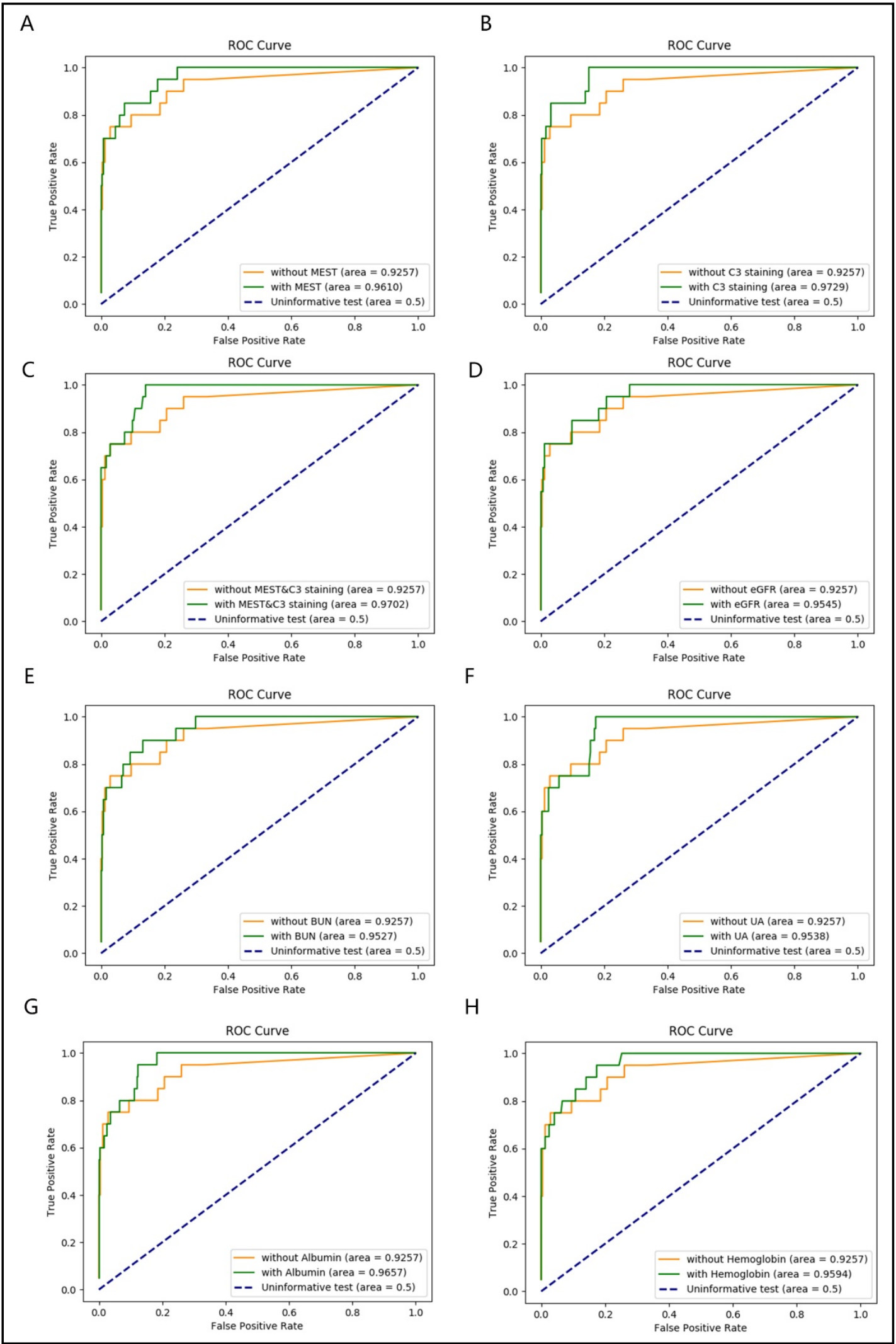

Fig. 4. Performance of Random Forest in ESRD prediction with different predictors. 


\section{Kidney Blood Pressure Research}

Kidney Blood Press Res 2018;43:1852-1864

\begin{tabular}{l|l}
\hline DOI: $10.1159 / 000495818$ & (c) 2018 The Author(s). Published by S. Karger AG, Basel
\end{tabular}

Published online: 8 December 2018

www.karger.com/kbr

1860

\section{Discussion}

In this study, we adopted a RF model to predict ESRD status in IgAN patients. We first evaluated the importance rankings of correlated predictive factors assessed by the RF model and then verified these factors by logistic regression analysis. The resulting important factors were further analysed in the predictive experiments. Specifically, we trained and evaluated a RF model using 6 predictors from the CDSS (www.IgAN.net). This RF model served as an initial model in our experiments. Next, we trained and evaluated the performances of several new RF models by integrating additional important factors that were obtained from the importance rankings, such as MEST scores, C3 staining, and eGFR. The new models outperformed the initial model for predicting ESRD. The improved predictive performance further verified the importance of these factors. Additionally, we hypothesized that the arithmetic method we adopted to conceive of the new model would not only be useful in Asian IgAN cohorts but could also be used to suit a wider population.

A well-established and widely accepted diagnostic and prognostic model can aid clinicians when informing the patients about a diagnosis and prognosis $[13,20]$ and thus helping clinicians make decisions regarding precise treatment and follow-up. Furthermore, predictors in the prognostic model can raise the importance and awareness of these factors during follow-up, thus enabling doctors to make consecutive and dynamic decisions.

Although it is simple to use one denominator, histological grading, which represents the IgAN pathology, as one input predictor, the potential use of MEST scores in prediction model was also discussed in the study of the ANN model [13]. Notably, most patients included in previously developed models were diagnosed before the publication of the Oxford classification; thus, it was not feasible to review all the registered data. The Oxford classification-MEST score, which includes four types of pathological features, namely, the mesangial hypercellularity score (M), endocapillary hypercellularity (E), segmental glomerulosclerosis (S), and tubular atrophy/interstitial fibrosis (T), has been shown to have independent value for predicting IgAN renal outcomes [21]. Several retrospective cohort studies have confirmed the prognostic value of MEST scores for predicting the clinical outcome of biopsy-proven IgAN patients $[8,22,23]$. In our cohort, $\mathrm{S}$ and $\mathrm{T}$ scores were significantly associated with renal survival in IgAN patients. Motivated by these studies, we integrated MEST scores in our prediction model to analyze whether this would improve prognostication. Our results indicated that the model including MEST scores showed better performance than the original model, as illustrated by the increased F-measure and AUC. Thus, MEST scores conveyed additional information regarding the prediction of poor renal outcomes in IgAN patients.

Various studies have suggested the predictive value of immunostaining features in patients with IgAN [24, 25]. Notably, C3 staining markedly improved the performance of the initial model in our study. We also found that the intensity of mesangial C3 staining was a potential predictor of IgAN progression, as assessed by logistic regression analysis and the RF importance score. Similarly, a recent study revealed that mesangial C3 deposition serves as an independent risk factor for renal survival in IgAN patients [26]. Including C3, other complement components present in the mesangial deposits are C4d, mannan-binding lectin(MBL), and MBL-associated serine proteases 1 and 2(MASP-1, 2) of the lectin pathway, and properdin and factor $\mathrm{H}$ of the alternative pathway [27,28]. Activation of the complement system in IgAN, specifically activation of the alternative and lectin pathways, has been shown to augment the inflammatory cascade and potentiate renal injury in IgAN $[27,29]$. On the other hand, a suit of complement components in serum, urine, or renal tissue may be potential biomarkers of IgAN. Performed as routine examinations in renal biopsies, increased C3 staining accompanied by decreased serum C3 levels significantly predicted poor renal outcome in IgAN patients [25]. Unfortunately, the baseline serum C3 levels measured in our study were not associated with renal outcomes. However, dynamically monitoring serum C3 levels could be more useful for evaluating complement system activity and disease prognosis. 


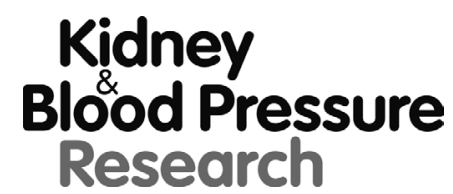
\begin{tabular}{l}
\hline Kidney Blood Press Res 2018;43:1852-1864 \\
\begin{tabular}{l|l} 
DOI: 10.1159/000495818 & c 2018 The Author(s). Published by S. Karger AG, Basel \\
Published onlıne: 8 December 2018 & www.karger.com/kbr
\end{tabular} \\
\hline
\end{tabular}

Liu et al.: ESRD Prediction in IgAN Patients via a Random Forest Model

Apart from classification schemes concerning renal pathology in IgAN, numerous predictive factors are controversial or not widely accepted: age at disease onset [30], gender, hyperuricaemia/ hypertriglyceridemia/[31-33], hypertension [11], eGFR[3, 4, 12], urinary markers $[34,35]$ and different immunogenetic markers (human leukocyte antigen (HLA), etc.). Among them, the eGFR is a rather strong predictor for IgAN prognosis [3, 4, 12]. We agree with Berthoux et al., who considered a reduction in eGFR as a continuum in the progression of IgAN as a renal function marker rather than a risk factor. However, IgAN becomes clinically evident during the late phase of chronic course, while patients often remain asymptomatic during the early phase. Therefore, leading time bias is an obvious influential factor when assessing the prognosis. Therefore, it is reasonable and rational to introduce a damage marker such as the eGFR to evaluate initial renal function at the time of diagnosis. By analysing the individual contribution of specific predictive factors via a machine-learning algorithm and logistic regression analysis, we found that the eGFR at the time of diagnosis made a considerable contribution to the poor prognosis of IgAN patients. Moreover, inclusion of this factor also improved the performance of the prognostic model.

The RF model has several advantages of its own. One obvious strengthen is that a RF is an ensemble of decision trees that provides a better interpretation than the ANN used in the CDSS for IgAN patients [13]. Researchers from the computer science community consider the ANN to be a black-box model. Interpreting the prediction results of a black-box model is quite difficult. Questions such as why the model outputs a specific prediction and how it makes use of the predictors to output the prediction cannot be answered with a black-box model. A model with a better interpretation can provide better insights into the roles of factors for predicting prognosis. In contrast to the ANN, the manner in which the RF model makes a prediction-regarding ESRD in IgAN patients can be presented by a flow diagram, as illustrated in the decision tree diagram (Fig. 1). As shown in the figure, each non-leaf node uses one factor to direct the samples to its child nodes. The leaf nodes denote prediction results. More details regarding training decision trees and RFs can be found in these papers $[14,36,37]$. Second, data splitting is not necessarily required to evaluate the performance of a RF model. All patients can be used to evaluate the performance of a RF model by utilizing the OOB error estimation scheme [17]. It has been empirically shown that the performance evaluated by the $\mathrm{OOB}$ error is as accurate as the the estimation of the performance obtained by the training/testing splitting scheme [18]. This advantage is extremely important for analysing the data from Asian patients due to the limited number of samples.

Another obvious strength of our study is the relatively large Asian population-based patient cohort with a specific follow-up time. However, the prognostic model needs further validation in other cohorts. In principle, every existing prognostic model should be tested on wider populations other than the patients in the dataset used to develop the model [38]. Another weakness is that we did not consider disease "natural history" or specific information regarding how the patients were treated. We provided data describing the use of drugs in our cohort. A similar proportion of RAS inhibitors and immunosuppressants were prescribed to the patients who progressed to ESRD and those who did not. On the other hand, there are no well-established recommendations or consensus for the evaluation and treatment of IgAN.

\section{Conclusion}

In summary, we explored the prognostic value of clinicopathological parameters for predicting ESRD status using a RF model. Our ultimate goal is to establish a complete computer-aided system that can be applied to predict ESRD status that best suits Asian IgAN patients. 


\section{Kidney Bloód Pressure Research}

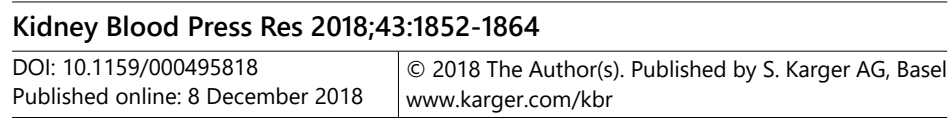

Liu et al.: ESRD Prediction in IgAN Patients via a Random Forest Model

\section{Disclosure Statement}

The authors have no conflicts of interest to declare.

\section{Acknowledgements}

This work was supported by grants from the National Natural Science Foundation of China (No. 81770714 and 81470947). Research idea and study design: Ye-xin Liu, Yan Zhang, Di Liu, Xia Tan and Hong Liu; data acquisition: Ye-xin Liu, Di Liu, Xia Tan, Xiao-fang Tang, Fan Zhang, Ming Xia; data analysis/interpretation: Ye-xin Liu, Yan Zhang, Fan Zhang, Ming Xia, Guo-chun Chen, Li-yu He, Le-tian Zhou, Xue-jing Zhu; statistical analysis: Ye-xin Liu, Yan Zhang, Di Liu, Xia Tan, Xiao-fang Tang, Guo-Chun Chen; supervision or mentorship: Liu Hong. Each author contributed content in drafting manuscript and all authors approved the final version of the manuscript. Ye-xin Liu and Hong Liu take responsibility that data in this study has been honestly reported.

\section{References}

1 Woo KT, Chan CM, Mooi CY, H. LC, Tan HK, Foo M, Lee GS, Anantharaman V, Lim CH, Tan CC, Lee EJ, Chiang GS, Tan PH, Boon TH, Fook-Chong S, Wong KS: The changing pattern of primary glomerulonephritis in Singapore and other countries over the past 3 decades. Clin Nephrol 2010;74:372-383.

-2 Lai KN, Tang SC, Schena FP, Novak J, Tomino Y, Fogo AB, Glassock RJ: IgA nephropathy. Nat Rev Dis Primers 2016;2:16001.

-3 Canetta PA, Kiryluk K, Appel GB: Glomerular diseases: emerging tests and therapies for IgA nephropathy. Clin J Am Soc Nephrol 2014;9:617-625.

4 Barbour SJ, Reich HN: Risk stratification of patients with IgA nephropathy. Am J Kidney Dis 2012;59:865873.

5 Liu H, Peng Y, Liu H, Liu Y, Yuan S, Liu F, Yang D, Chen X, He L, Fu M, Shao J, Yang L: Renal biopsy findings of patients presenting with isolated hematuria: disease associations. Am J Nephrol 2012;36:377-385.

6 Li PK, Ho KK, Szeto CC, Yu L, Lai FM: Prognostic indicators of IgA nephropathy in the Chinese--clinical and pathological perspectives. Nephrol Dial Transplant 2002;17:64-69.

7 Le W, Liang S, Hu Y, Deng K, Bao H, Zeng C, Liu Z: Long-term renal survival and related risk factors in patients with IgA nephropathy: results from a cohort of 1155 cases in a Chinese adult population. Nephrol Dial Transplant 2012;27:1479-1485.

-8 Zhu X, Li H, Liu Y, You J, Qu Z, Yuan S, Peng Y, Liu F, Liu H: Tubular atrophy/interstitial fibrosis scores of Oxford classification combinded with proteinuria level at biopsy provides earlier risk prediction in $\lg A$ nephropathy. Sci Rep 2017;7:1100.

9 D'Amico G: Natural history of idiopathic IgA nephropathy: role of clinical and histological prognostic factors. Am J Kidney Dis 2000;36:227-237.

10 Bartosik LP, Lajoie G, Sugar L, Cattran DC: Predicting progression in IgA nephropathy. American Journal of Kidney Diseases 2001;38:728-735.

11 Berthoux F, Mohey H, Laurent B, Mariat C, Afiani A, Thibaudin L: Predicting the risk for dialysis or death in IgA nephropathy. J Am Soc Nephrol 2011;22:752-761.

12 Goto M, Wakai K, Kawamura T, Ando M, Endoh M, Tomino Y: A scoring system to predict renal outcome in IgA nephropathy: a nationwide 10-year prospective cohort study. Nephrol Dial Transplant 2009;24:30683074.

13 Pesce F, Diciolla M, Binetti G, Naso D, Ostuni VC, Di Noia T, Vagane AM, Bjorneklett R, Suzuki H, Tomino Y, Di Sciascio E, Schena FP: Clinical decision support system for end-stage kidney disease risk estimation in IgA nephropathy patients. Nephrol Dial Transplant 2016;31:80-86.

14 Breiman L: Random Forests. Machine Learning 2001;45:5-32. 


\section{Kidney \\ Blood Pressure Research}

Kidney Blood Press Res 2018;43:1852-1864

\begin{tabular}{l|l}
\hline DOI: $10.1159 / 000495818$ & (c) 2018 The Author(s). Published by S. Karger AG, Basel
\end{tabular}

Published online: 8 December 2018 www.karger.com/kbr

Liu et al.: ESRD Prediction in IgAN Patients via a Random Forest Model

15 Imai E, Horio M, Nitta K, Yamagata K, Iseki K, Tsukamoto Y, Ito S, Makino H, Hishida A, Matsuo S: Modification of the Modification of Diet in Renal Disease (MDRD) Study equation for Japan. Am J Kidney Dis 2007;50:927-937.

16 Working Group of the International IgA Nephropathy Network and the Renal Pathology Society, Roberts IS, Cook HT, Troyanov S, Alpers CE, Amore A, Barratt J, Berthoux F, Bonsib S, Bruijn JA, Cattran DC, Coppo R, D'Agati V, D'Amico G, Emancipator S, Emma F, Feehally J, Ferrario F, Fervenza FC, Florquin S, et al.: The Oxford classification of IgA nephropathy: pathology definitions, correlations, and reproducibility. Kidney Int 2009;76:546-556.

17 Breiman L: Bagging Predictors. Machine Learning 1996;24:123-140.

18 Breiman L: Out-of-bag estimation. University of California. URL: https://www.stat.berkeley.edu/users/ breiman/OOBestimation.pdf.

19 Zhang F, Liu H, Liu D, Liu Y, Li H, Tan X, Liu F, Peng Y, Zhang H: Effects of RAAS Inhibitors in Patients with Kidney Disease. Curr Hypertens Rep 2017;19:72.

20 Yang Y, Luo M, Xiao L, Zhu XJ, Wang C, Fu X, Yuan SG, Xiao F, Liu H, Dong Z, Liu FY, Sun L: Exploration of pathological prediction of chronic kidney diseases by a novel theory of bi-directional probability. Sci Rep 2016;6:32151.

21 Working Group of the International IgA Nephropathy Network and the Renal Pathology Society, Cattran DC, Coppo R, Cook HT, Feehally J, Roberts IS, Troyanov S, Alpers CE, Amore A, Barratt J, Berthoux F, Bonsib S, Bruijn JA, D'Agati V, D'Amico G, Emancipator S, Emma F, Ferrario F, Fervenza FC, Florquin S, et al.: The Oxford classification of IgA nephropathy: rationale, clinicopathological correlations, and classification. Kidney Int 2009;76:534-545.

-22 Barbour SJ, Espino-Hernandez G, Reich HN, Coppo R, Roberts IS, Feehally J, Herzenberg AM, Cattran DC, Oxford Derivation NAV, Consortia V, Oxford Derivation North American V, Consortia V: The MEST score provides earlier risk prediction in lgA nephropathy. Kidney Int 2016;89:167-175.

23 Herzenberg AM, Fogo AB, Reich HN, Troyanov S, Bavbek N, Massat AE, Hunley TE, Hladunewich MA, Julian BA, Fervenza FC, Cattran DC: Validation of the Oxford classification of IgA nephropathy. Kidney Int 2011;80:310-317.

24 Nakagawa H, Suzuki S, Haneda M, Gejyo F, Kikkawa R: Significance of glomerular deposition of C3c and C3d in IgA nephropathy. Am J Nephrol 2000;20:122-128.

-25 Kim SJ, Koo HM, Lim BJ, Oh HJ, Yoo DE, Shin DH, Lee MJ, Doh FM, Park JT, Yoo TH, Kang SW, Choi KH, Jeong HJ, Han SH: Decreased circulating C3 levels and mesangial C3 deposition predict renal outcome in patients with IgA nephropathy. PLoS One 2012;7:e40495.

26 Caliskan Y, Ozluk Y, Celik D, Oztop N, Aksoy A, Ucar AS, Yazici H, Kilicaslan I, Sever MS: The Clinical Significance of Uric Acid and Complement Activation in the Progression of IgA Nephropathy. Kidney Blood Press Res 2016;41:148-157.

27 Maillard N, Wyatt RJ, Julian BA, Kiryluk K, Gharavi A, Fremeaux-Bacchi V, Novak J: Current Understanding of the Role of Complement in IgA Nephropathy. J Am Soc Nephrol 2015;26:1503-1512.

-28 Roos A, Rastaldi MP, Calvaresi N, Oortwijn BD, Schlagwein N, van Gijlswijk-Janssen DJ, Stahl GL, Matsushita M, Fujita T, van Kooten C, Daha MR: Glomerular activation of the lectin pathway of complement in IgA nephropathy is associated with more severe renal disease. J Am Soc Nephrol 2006;17:1724-1734.

29 Roos A, Bouwman LH, van Gijlswijk-Janssen DJ, Faber-Krol MC, Stahl GL, Daha MR: Human IgA activates the complement system via the mannan-binding lectin pathway. J Immunol 2001;167:2861-2868.

-30 Donadio JV, Bergstralh EJ, Grande JP, Rademcher DM: Proteinuria patterns and their association with subsequent end-stage renal disease in IgA nephropathy. Nephrol Dial Transplant 2002;17:1197-1203.

-31 Shi Y, Chen W, Jalal D, Li Z, Chen W, Mao H, Yang Q, Johnson RJ, Yu X: Clinical outcome of hyperuricemia in IgA nephropathy: a retrospective cohort study and randomized controlled trial. Kidney Blood Press Res 2012;35:153-160.

-32 Trevino-Becerra A: Hypertriglyceridaemia and hyperuricaemia in IgA nephropathy. Nephrol Dial Transplant 2001;16:639.

-33 Syrjanen J, Mustonen J, Pasternack A: Hypertriglyceridaemia and hyperuricaemia are risk factors for progression of IgA nephropathy. Nephrol Dial Transplant 2000;15:34-42. 


\section{Kidney Blood Pressure Research}

34 Jiang WL, Peng YM, Liu YH, Liu H, Chen GC, Xu XQ, Zhu XJ, Liu FY: Evaluation of renal clinicopathological changes in IgA nephropathy by urinary podocytes excretion and podocalyxin expression. Ren Fail 2012;34:821-826.

35 Wang H, Zheng C, Lu Y, Jiang Q, Yin R, Zhu P, Zhou M, Liu Z: Urinary Fibrinogen as a Predictor of Progression of CKD. Clin J Am Soc Nephrol 2017;12:1922-1929.

36 Breiman L, Friedman J, Stone CJ, Olshen RA: Classification and Regression Trees, UK, Taylor \& Francis, 1984.

-37 Salzberg, SL: Book Review: C4.5: programs for machine learning by J. Ross Quinlan. Morgan Kaufmann Publishers Inc., 1993. Mach Learn 1994;16:235.

-38 Tripepi G, Heinze G, Jager KJ, Stel VS, Dekker FW, Zoccali C: Risk prediction models. Nephrol Dial Transplant 2013;28:1975-1980. 